\title{
The use of indexes evaluating the adherence to the Mediterranean diet in epidemiological studies: a review
}

\author{
Anna Bach ${ }^{1,3, *}$, Lluís Serra-Majem ${ }^{1,2}$, Josep L Carrasco ${ }^{3}$, Blanca Roman ${ }^{1}$, Joy Ngo ${ }^{1}$, \\ Isabel Bertomeu ${ }^{1}$ and Biel Obrador ${ }^{4}$ \\ ${ }^{1}$ Foundation for the Advancement of the Mediterranean Diet, University of Barcelona Science Park, Spain: \\ ${ }^{2}$ Department of Clinical Sciences, University of Las Palmas de Gran Canaria, Spain: ${ }^{3}$ Biostatistics, Department of \\ Public Health, University of Barcelona, Spain: ${ }^{4}$ Faculty of Biology, University of Barcelona, Spain
}

\begin{abstract}
The purpose of this paper is to review some of the methods that several epidemiological studies use to evaluate the adherence of a population to the Mediterranean diet pattern. Among these methods, diet indexes attempt to make a global evaluation of the quality of the diet based on a traditional Mediterranean reference pattern, described as a priori, general and qualitative. The Mediterranean diet indexes, hence, summarise the diet by means of a single score that results from a function of different components, such as food, food groups or a combination of foods and nutrients. The reviewed evaluation methods can be classified into three categories depending on the way they are calculated: (1) those based on a positive or negative scoring of the components, (2) those that add or substract standardised components, and (3) those that are based on a ratio between components.

Dietary scores have been used to explore the multiple associations between the Mediterranean diet, as an integral entity, and health parameters such as life expectancy or the incidence of obesity, cardiovascular diseases and some types of cancers. Moreover, these indexes are also useful tools to measure food consumption trends and to identify the involved factors, as well as to develop comprehensive public health nutrition recommendations.

A more precise and quantitative definition of the Mediterranean diet is required if the adherence to such a dietary pattern is intended to be more accurately measured. Other aspects of the Mediterranean diet indexes should also be taken into account, like the inclusion of typical Mediterranean foods such as nuts and fish and the validation of the dietary pattern approach by using biomarkers.
\end{abstract}

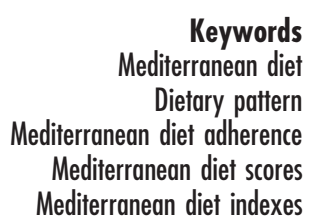

Historically, in epidemiological studies, the majority being observational, diets consumed by Mediterranean populations have been of interest due to the observation that adults who lived near the Mediterranean Sea had one of the lowest incidences in chronic diseases in the world and one of the highest life expectancies ${ }^{1,2}$. In the last few decades, numerous associations have been postulated between health status and the Mediterranean diet (MD) and some of its components. For example, it has been suggested that variants of this diet may improve the prognosis in coronary heart disease patients ${ }^{3}$, that some aspects of the Mediterranean diet pattern (MDP) may protect against the development of diabetes mellitus type $\mathrm{II}^{4}$, hypertension ${ }^{5}$, embolisms ${ }^{6}$ and osteoporosis ${ }^{7}$. Additionally, a beneficial effect is suggested with some cancers, such as breast cancer, stomach cancer, colorectal cancer and prostate cancer ${ }^{8}$.

The traditional MD refers to the dietary pattern in the Mediterranean olive grove areas at the beginning of the 1960s, during the post World War II recovery period but before these areas were influenced by fast-food culture? $^{9}$. However, the Mediterranean diet is not a homogeneous model within the Mediterranean area. It presents regional variations derived from the same dietary pattern, influenced by various factors, such as socio-cultural, religious and economic determinants, to name a few.

The MDP has been defined in several international scientific meetings $s^{3,10,11}$ as varied, not very caloric and based on fresh, local and seasonal products, when possible. This pattern is represented in the Mediterranean Diet Pyramid, a graphic indication that daily intake should be mainly composed of foods of vegetable origin: cereals, fruits, vegetables, legumes and nuts are located at the base of the pyramid. And, with a decreasing intake, in frequency and quantity, in a step up in the pyramid: dairy products, potatoes, poultry, eggs: and on the top, to consume occasionally, sweets, meat and its derivatives. 
Other common characteristics are the use of olive oil as the main source of fat, the presence of moderate wine intake at meals and a frequent intake of fish, based on the proximity to the sea.

Most of the scientific knowledge used as evidence for the creation of food guidelines is based on associations between foods or nutrients and the incidence of certain diseases ${ }^{12,13}$. But it has not been until the 21st century when scientific studies started exploring food patterns in health and disease ${ }^{14}$. Until fairly recently there were no systems to evaluate and adequately summarise all the information regarding food patterns ${ }^{15}$. To analyse food patterns there are two approximations: developing food indexes, i.e. food scores according to the intake of certain foods; or deriving patterns via multi-variant analysis by means of a factorial analysis, principal component analysis or cluster analysis ${ }^{16}$.

The purpose of this paper is to review some of the methods that several epidemiological studies have used to evaluate the adherence of a population to the MDP. The Mediterranean diet indexes attempt to make a global evaluation of the quality of the diet based on a traditional Mediterranean 'reference' pattern, described as 'a priori', being general and qualitative. The Mediterranean diet indexes, hence, summarise the diet by means of a single score that results from a function of different components, such as food, food groups or a combination of foods and nutrients. These components are previously selected based on prior knowledge or scientific evidence, this approach thus being an 'a priori approximation'.

\section{Material and methods}

An English and Spanish literature search has been done through databases (MEDLINE; NCBI, Bethesda, MD, USA), cited references in related publications, and proceedings of the biannual Barcelona International Congress on the Mediterranean Diet, in order to examine publications on Mediterranean diet adherence indexes. Keywords included were: Mediterranean diet, dietary pattern, Mediterranean diet adherence, Mediterranean diet scores, and Mediterranean diet indexes.

\section{Results}

The earlier general diet quality indexes generated the initiative to create the current Mediterranean diet indexes. The reviewed evaluation methods can be classified into three categories depending on the way they are calculated: (1) those based on a positive or negative scoring of the components, (2) those that add or substract standardized components, and (3) those that are based on a ratio of components. All results are summarised in Table 1.

\section{Index by positive or negative component scoring}

The Mediterranean Diet Score

The 'Mediterranean Diet Score' (MDS) was created to measure the adherence gradient to the Greek MDP ${ }^{17}$. The MDS is the most extensively used index due to its ease of application, and many variants have been created for the evaluation of multiple diet-health relationships.

The Traditional Greek MD was simplified into eight components to define the MDS- ${ }^{17}$ : (1) High ratio of monounsaturated:saturated fat, (2) Moderate alcohol intake, (3) High legume intake, (4) High intake of grains (including bread and potatoes), (5) High fruit intake, (6) High vegetable intake, (7) Low intake of meat and meat products and (8) Moderate intake of milk and dairy products.

The MDS-1 was based on assigning a score from 0 to 1 according to the daily intake of the eight components. In general, the medians of the sample, specific for sex, were used as cut-off points ${ }^{18}$ and grams per day were used as the intake measurement ${ }^{17}$. A subject received a point if his intake was over the sample median for a protective component (vegetables, fruits, etc.) and below the median for non-protective components (dairy products, meat, etc.). In the case of alcohol (except when specified) 1 point was scored for males if their consumption was within 10 and $50 \mathrm{~g} /$ day, and within 5 and $25 \mathrm{~g} /$ day for women. If all the characteristics of the diet were incorporated, the highest score was obtained and reflected a greater adherence to the MD. Therefore, the MDS-1 usually ranged from 0 (minimal adherence) to 8 (maximum adherence) if the index had eight components. Generally, a score of 4 or more was associated with satisfactory MDP adherence and better health implications $^{17,19}$. In most studies, intake was adjusted for calories consumed, $2500 \mathrm{kcal}$ for men and $2000 \mathrm{kcal}$ for women, so the estimations would be independent of the variations present in energy intake.

Greek studies. In the first study of this series, Greek researchers prospectively evaluated the role of the diet in longevity ${ }^{17}$. The cohort study included 182 subjects, all of them older than 70, living in three rural towns of Greece. It was observed that the adherence to the Mediterranean diet (MDS $\geq 4$ ) significantly affected elderly life expectancy. Increasing 1 point on the MDS- 1 reduced the risk of total mortality by $17 \%$ and by $50 \%$ with an increase of 4 points.

Subsequently, between 1994 and 1999, another cohort study was carried out in a sample of 22043 adults $^{20}$. This showed that a greater adherence to the traditional Mediterranean diet was significantly associated with a reduced total mortality for coronary heart disease and cancer; with an increment of 2 points on the MDS-2 corresponding to a reduction of $25 \%$ of the above mentioned mortality. The reduction was stronger in coronary mortality than in cancer mortality. In this study, an MDS-2 of 10 components was used, differing from the 


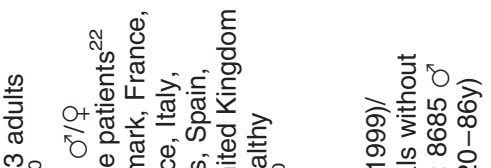

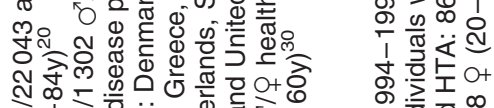

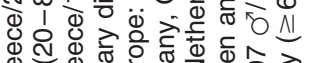

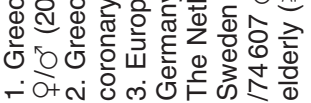

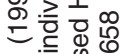
我. $\stackrel{\infty}{\infty} \frac{\mathrm{O}}{\mathrm{O}+}$

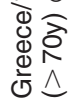

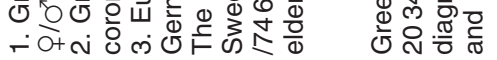<smiles>COC1CC1</smiles>

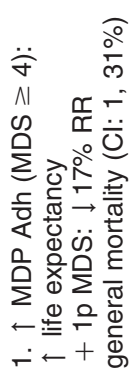

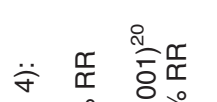

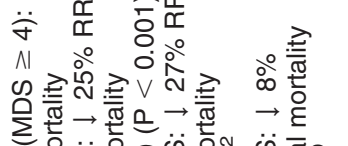
둫

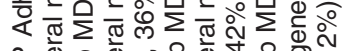

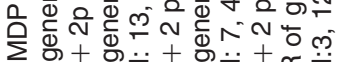

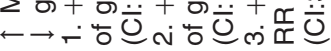

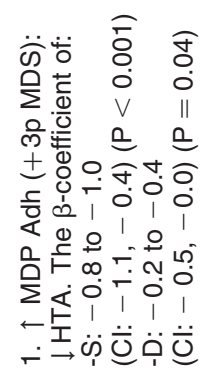

푸요

in $\div$

$\stackrel{\square}{\underline{0}}$

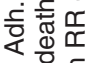

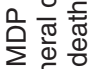

$\stackrel{\square}{\rightarrow} \stackrel{\circ}{\rightarrow}$

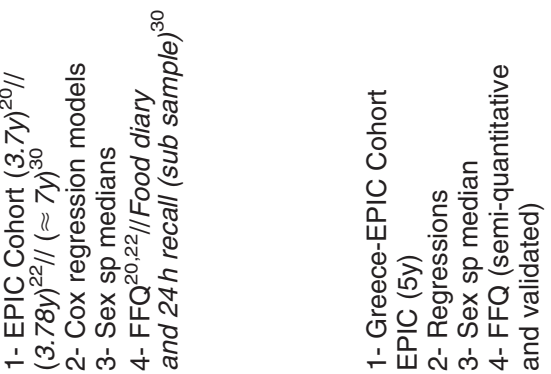

นᄂ $\frac{0}{\frac{\pi}{0}}$

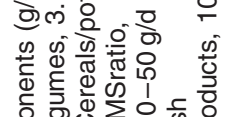

$\overbrace{\Sigma}^{0}$

竞 항

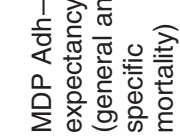

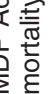

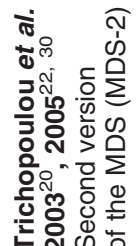

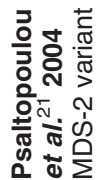

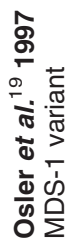


Indexes evaluating adherence to the Mediterranean diet

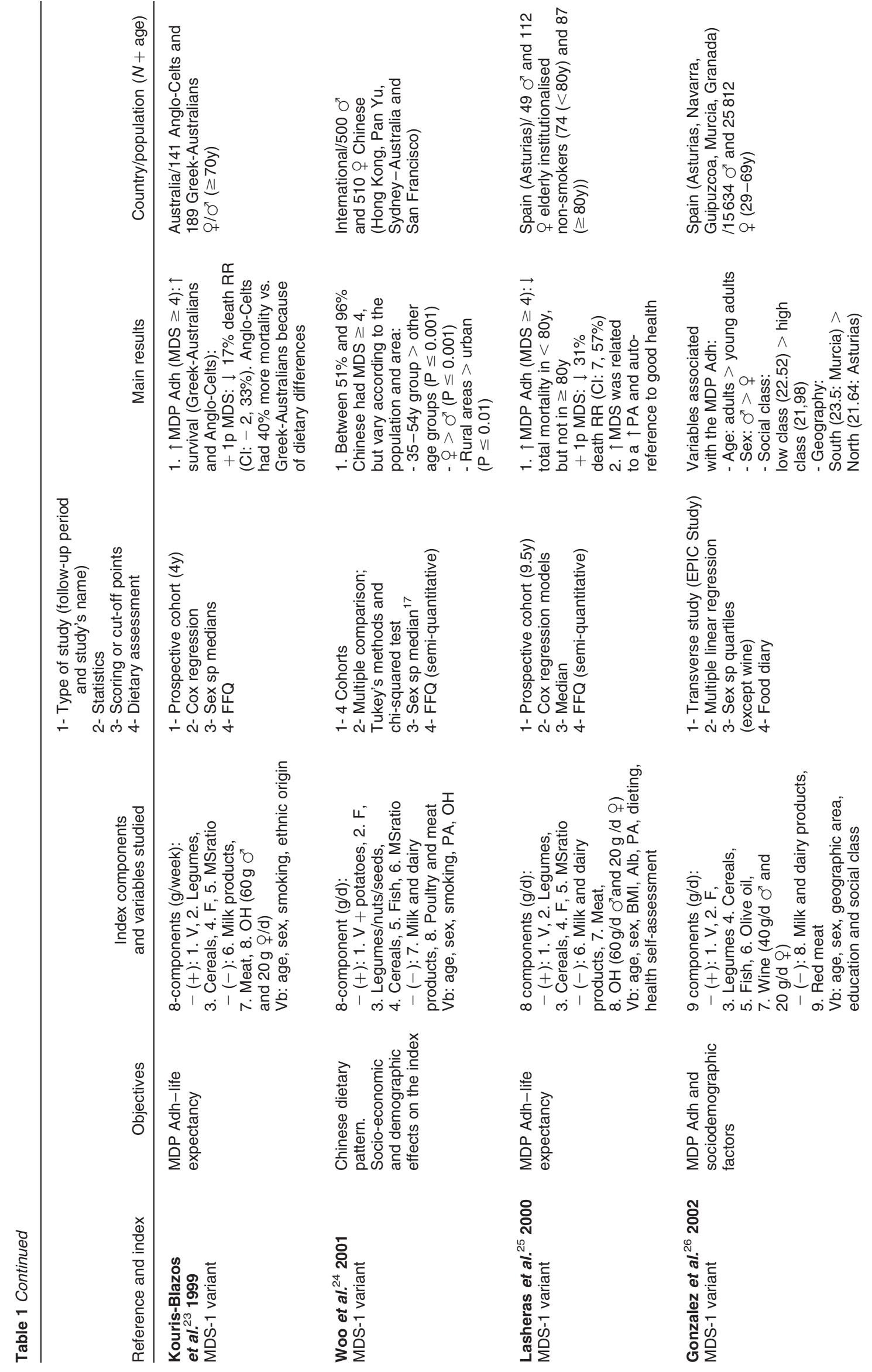


$\ddot{\widetilde{\sigma}}$

坚

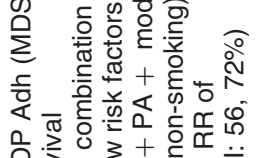

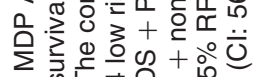

‘

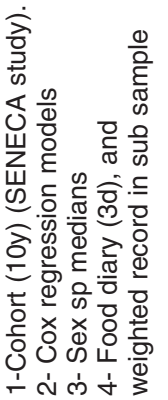

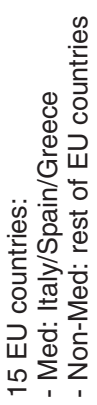

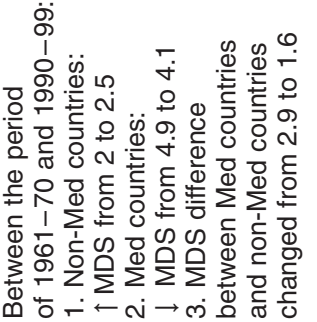

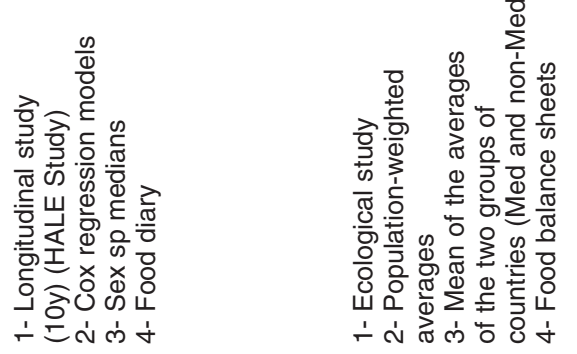
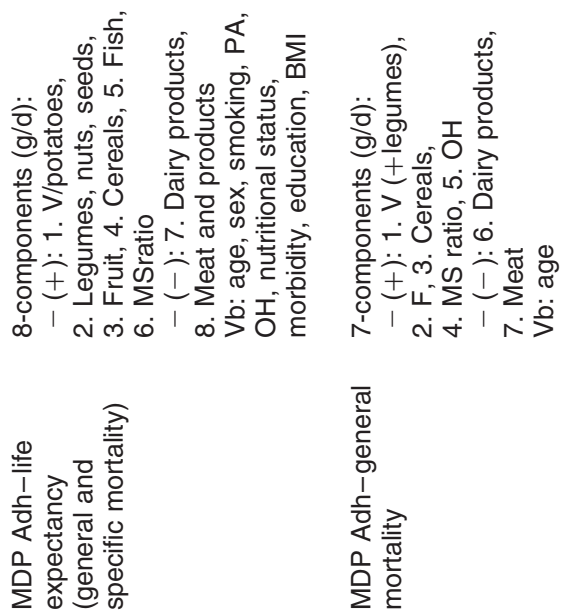

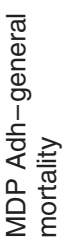

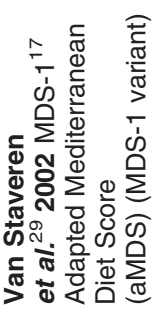

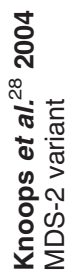

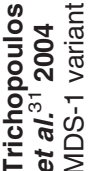




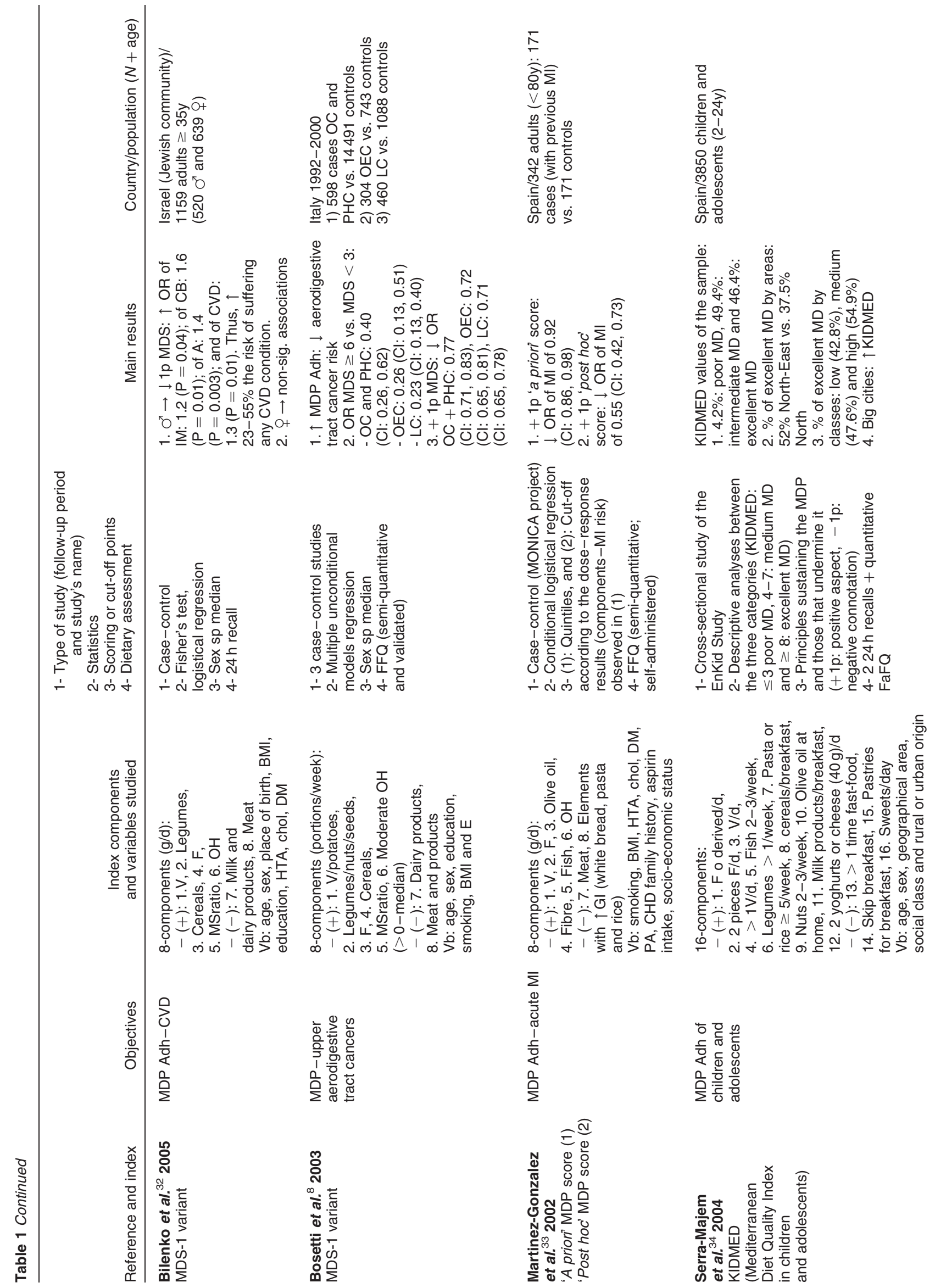


A Bach et al.
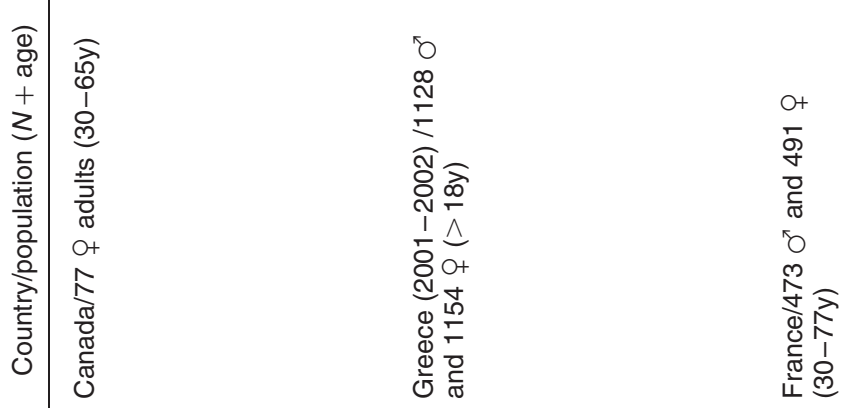

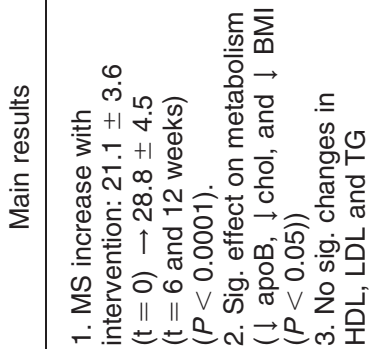

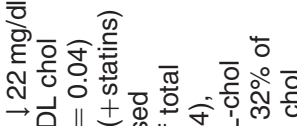

î

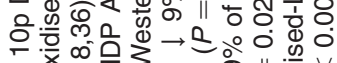

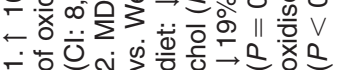

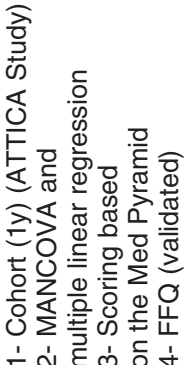

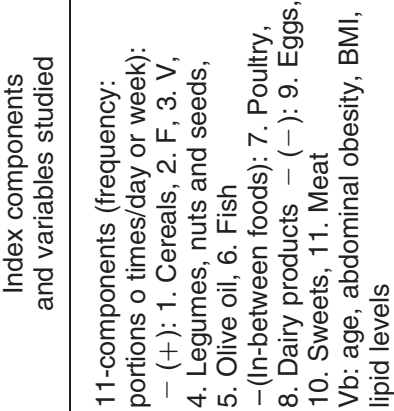

.. $\frac{\infty}{\pi}$

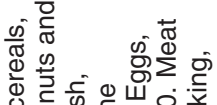
ब.

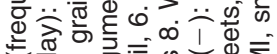
至

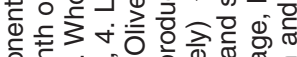

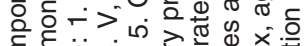

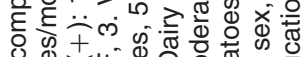

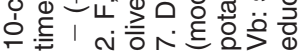
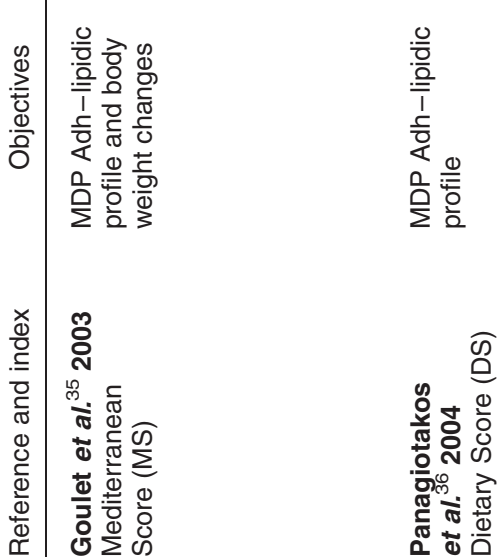
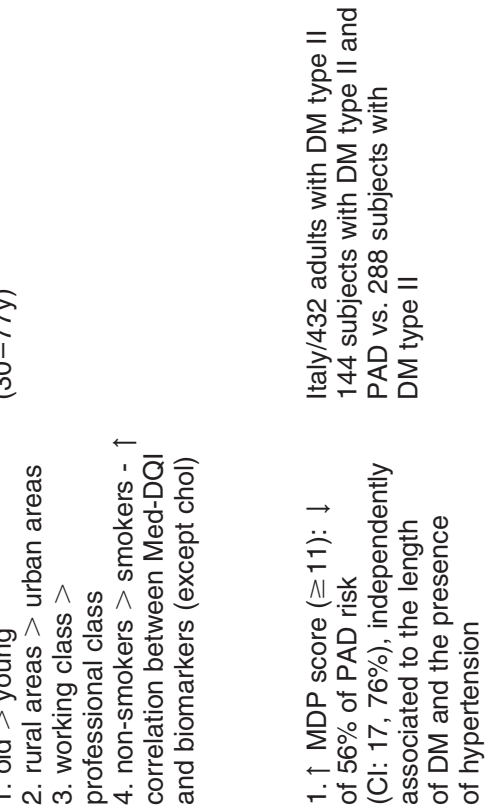
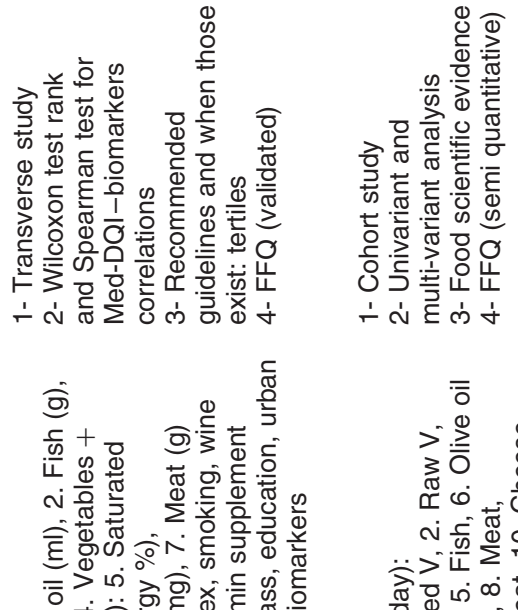

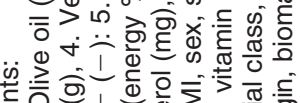
कㄴ. 누ㅇㅝㅔ

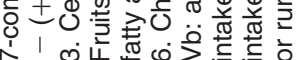
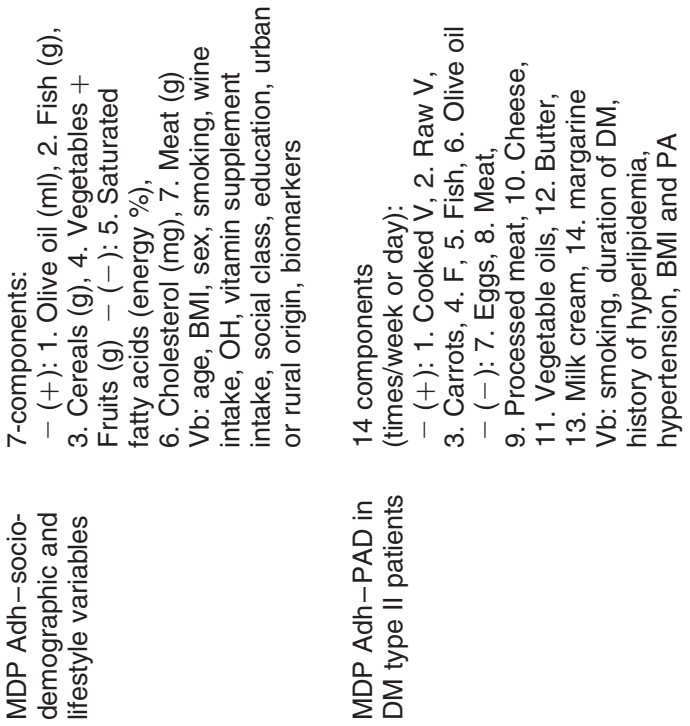

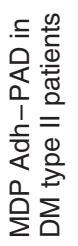

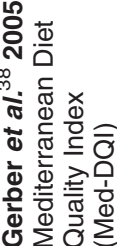




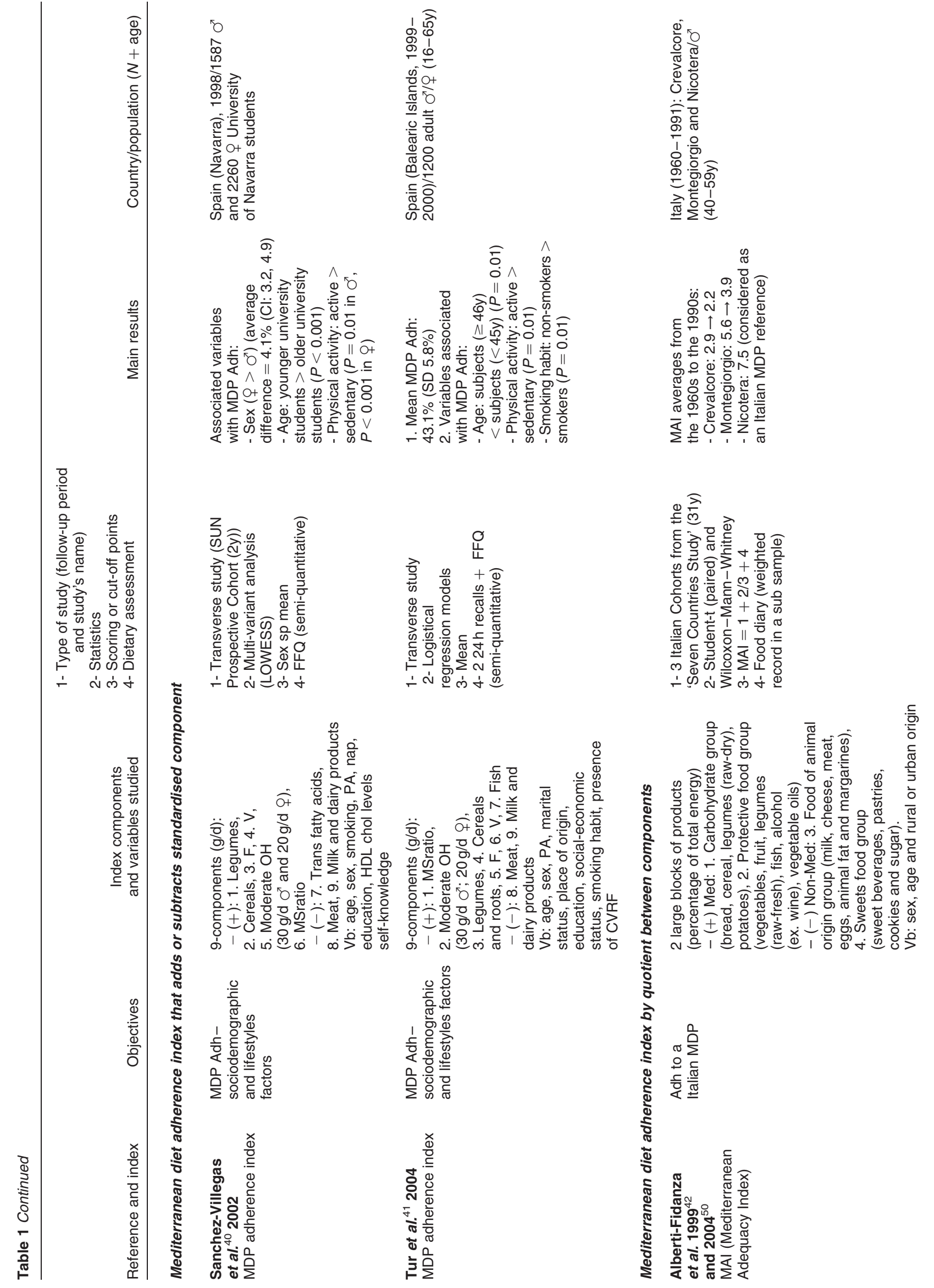


๓

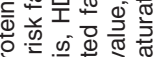

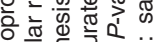

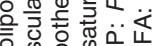

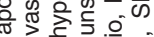

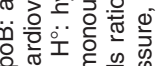

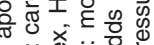

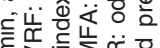

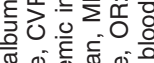
क 娄署

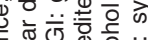

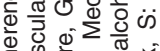

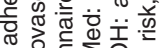

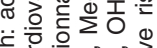

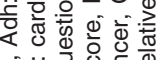
ำ 항

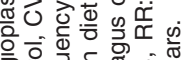

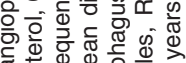
ब

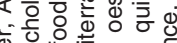

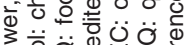

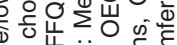
क्षे क्षे

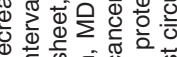

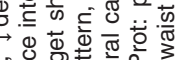

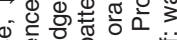

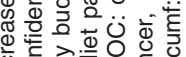

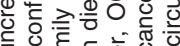

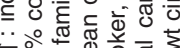

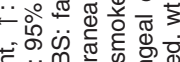

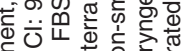

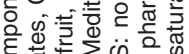

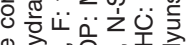

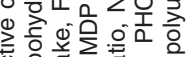
递要

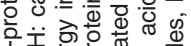

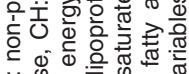

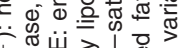

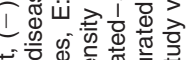
式要边

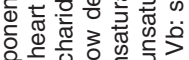

initial 8-point MDS-1, as the second version incorporated fish and moderate poultry intake.

The Greek branch of the European Prospective Investigation into Cancer and Nutrition (EPIC) cohort explored the association between the MDP and olive oil for hypertension, since hypertension can give way to different forms of cardiovascular disease ${ }^{21}$. A ninecomponent variant of the MDS was applied, finding it inversely related to systolic and diastolic blood pressure, after socio-demographic and anthropometric covariates were controlled. Although cereal intake, a component considered protective, was positively associated with high arterial blood pressure.

Once again, based on the Greek branch of the EPIC cohort, a variant of the MDS was applied to a Greek sample of 1302 coronary disease patients, observing that those who presented a greater MDP adherence had a reduced risk of general mortality ${ }^{22}$. Specifically, with a 2point increase in the score the relative risk of general mortality was reduced by $27 \%$, and by $31 \%$ in regards to coronary heart disease.

Danish studies. As part of the Euronut Survey in Europe on Nutrition and the Elderly, Olser et al. ${ }^{19}$ via a Concerted Action (SENECA) examined the influence of the MDS on general survival in a cohort of 202 Danes of advanced ages, with a 6-year follow-up ${ }^{19}$. Data regarding food intake was obtained by a 3-day dietary diary and a food frequency questionnaire. The MDS was comprised of seven components, and as a reflection of the Danish food patterns, vegetables and legumes were combined into one component. An increase of 1 point on the score predicted a reduction by $21 \%$ of global mortality.

Australian studies. A cohort study of a similar design to the above mentioned studies, with a sample of 141 AngloCelts and 189 Greco-Australians, was realised in Melbourne $^{23}$. There was a double objective; on one hand to evaluate if the rural Greek results could be replicated in an urban Australian environment and on the other hand, to analyse if the benefits of the Mediterranean diet were applicable to non-Mediterranean populations. The eating habits of the participants were evaluated with extensive and validated frequency questionnaires. The results were compatible with the hypothesis that a high score, i.e. greater or equal to 4 , was associated with longer survival. In this study, 153 (81\%) Greco-Australians and 39 (28\%) Anglo-Celts gathered four or more Mediterranean diet characteristics. An increase of 1 point on the score supposed a reduction of $17 \%$ in general mortality.

Chinese studies. The Chinese diet shares some characteristics with the Mediterranean diet, such as a high intake of vegetables and fruit and a low intake of meat. Chinese researchers aimed to determine if dietary habits of some Chinese populations were similar to those of the MDP, with an MDS adapted to the Chinese $\operatorname{diet}^{24}$. 
The score was calculated for 1010 Chinese living in four very diverse geographic regions: Hong Kong, a rural town near Pan Yu in southern China, Sydney (Australia) and San Francisco (USA). The results indicated that the majority of the Chinese population, living in China or elsewhere, had dietary patterns similar to the Mediterranean, and achieved in the majority of cases higher MDS scores as compared with Trichopoulou et al.'s ${ }^{17}$ Greek population. Middleaged individuals (35-54 years) obtained a higher score than those in other age groups, as did women when compared to men. Moreover, rural populations showed greater adherence due to the ease of maintaining the dietary pattern.

Spanish studies. A prospective cohort study was carried out during 9 years in 161 Spanish nursing home residents, 65 years old or older and non-smokers ${ }^{25}$. An increase in 1 point on the score was associated with a significant reduction in mortality by $31 \%$ in individuals younger than 80 years old. However, no significant associations were found in populations older than 80 years of age.

A transverse study examined the influence of demographic and social variables on the adherence to MDP in 15634 women and 25812 men aged 29 to 69, who were volunteers to the EPIC-Spain Study ${ }^{26}$. A modified version of the initial MDS- $1^{17}$ was used. Each of the nine components of the score received 1 to 4 points based on the quartiles of intake (calories adjusted), except in the case of wine, where moderate intake was used as a cut-off point. No variations in adherence to the MDP based on educational level were found, but small differences regarding social status were seen. Less adherence was observed in young adults and in women, and was slightly higher in southern areas as compared to northern Spain.

An MDS variant was applied to study the relationship between the Mediterranean diet and obesity ${ }^{27}$. The index was calculated with the exception of red wine consumption, according to the tertile distribution of intake. The total score that could be obtained ranged from 9 to 27 points. Increasing the score by 5 units, body mass index (BMI) decreased significantly in men and in women, controlling potentially confounding variables such as sociodemographic and lifestyle factors (i.e. physical activity). Individuals in the upper quartile with respect to those in the lower quartile of the score had 39\% less risk of obesity, for both men and women.

European studies. The Healthy Aging: a Longitudinal study in Europe (HALE) project is a prospective study that followed men and women of advanced age during 10 years in 11 countries around Europe to investigate the association between diet and lifestyle factors with mortality due to coronary, cardiovascular and cancer causes $^{28}$. The HALE project included participants proceeding from two studies: SENECA and the Finland, Italy, the Netherlands, Elderly study (FINE) ${ }^{28,29}$. The MDS was applied to these data, taking into account some modifications with respect to the original MDS ${ }^{17}$. Potatoes were added to the vegetable group, fish was added as an independent category and alcohol was not included in the score. Sex-specific median intakes were adopted as cut-off points. To evaluate the association between mortality and lifestyle variables, a low risk group was established having the following characteristics: high dietary score, nonsmoker, moderate drinker and physically active. The adherence to the MD was associated with $22 \%$ less risk of general mortality, being physically active and a nonsmoker with 37\% and 35\% less risk, respectively. The combination of the four protective factors reduced general mortality by $60-64 \%$, supporting the hypothesis that the participants who followed an MD and maintained healthy lifestyle habits had less general and specific mortality, even in ages 70 to 90 years old.

From the final SENECA European study, where 1507 men and 832 women of advanced age from 12 European countries participated, predictive values of dietary patterns in survival during 10 years were evaluated using the original MDS (MDS-1) and an adaptation (aMDS) ${ }^{29}$. The adaptation consisted of varying the cut-off point of certain components: the optimal intake of dairy products was considered as an interquartilic range for men and women, the optimal intake of meat and poultry in women was set as below the 75 th percentile and the maximum alcohol intake in women was also set at the 75th percentile. The application of the MDS-1 did not yield a significant positive association between diet and life expectancy. On the other hand, the aMDS, although not contributing significant results, did show a clear tendency that a favourable score was related to higher survival.

Recently, the EPIC-Elderly prospective cohort study evaluated the relationship between the 'modified' Mediterranean diet and the survival of 74607 individuals aged 60 years or more in nine European countries ${ }^{30}$. The adherence to the MDP was measured through the MDS- $2^{20}$, in which a lipid ratio was incorporated where the polyunsaturated fatty acids appear in the numerator, so that the index could be applied to non-Mediterranean European countries. It was found that a greater adherence to the MDP was associated with a significant reduction in general mortality. An increment of 2 points entailed a reduction of $8 \%$ of relative risk of mortality and $7 \%$ when the exposition factors were calibrated between countries.

Also within the European context, differences in following the MDP were studied between Mediterranean countries and non-Mediterranean countries based on FAO's Food Balance Sheets ${ }^{31}$. During the study period (1961-1970 and 1990-1999) it was observed that nonMediterranean countries presented an increase of a sevencomponent mean score from 2 to 2.5 , which reflected an increase of fruit, vegetable and vegetable fat consumption. In contrast, the diet of Mediterranean countries was negatively affected by the increased intake of meat and animal fat, as shown by a reduction in MDS mean from 4.9 
to 4.1. Therefore, the differences in the index score between the two groups of countries were reduced, this being associated with the reduction in the differences in general mortality observed for the two groups.

Israeli studies. The MDS was applied to 1159 Jewish people ${ }^{32}$, finding that there were relatively low levels of adherence to the pattern (less than 20\%). A strong association between the low MDP adherence (MDS $\leq 4$ ) and cardiovascular disease was found only in men. A reduction of 1 point in the MDS was related to a $23-55 \%$ increased risk for cardiovascular disease.

Italian studies. In Italy, it was observed that the Mediterranean diet favourably decreased the risk of suffering aerodigestive cancers (oral, oesophagus, pharynx, and larynx) in three case-control studies, with a total sample of 4684 individuals, where the original MDS- $1^{17}$ was applied ${ }^{8}$. Therefore, an increase of 1 point on the score meant a reduced risk of $23 \%$ in the case of oral and pharynx cancer, 28\% in oesophageal cancer and 29\% in larynx cancer.

\section{'Post hoc' Mediterranean diet pattern score}

To understand if the MDP plays a protective role in reinfarction and cardiovascular death, a 'post hoc' index was created based on 'a priori' defined MDP ${ }^{33}$. The ' $a$ priori' index was made up of eight components, where apart from foods, such as those with high glycaemic index, nutrients could also be found. According to the quintile distribution by the intake of each component (adjusted by energy) a score was assigned from 1 to 5 for each element. In the case of protective components 1 point was assigned to the lower quintile and 5 points to the upper quintile, and for the non-protective components, the scoring was inverted. This way, each participant had a total score that ranged from 8 to 40. In the 'post hoc' index a single cut-off point was used for each component based on the 'previous' results of the dose-response associations between the intake of each component and the risk of suffering a second myocardial infarction. For the majority of index components, individuals in the second quintile had a major reduction of risk in comparison to those in the first quintile, but no significant differences between Q2 and Q5 were observed. Therefore, with the 'post hoc' index if the participant consumed more than this value (Q2) a point was assigned for each component, thus obtaining a score between 0 and 8 . The results of both indexes indicated that when the score increased, the odds ratio for myocardial infarctions was significantly reduced.

\section{KIDMED Index}

The KIDMED Index was a Mediterranean diet quality index constructed to evaluate the food habits of a population of 3850 Spanish children and adolescents aged between $2-24$ years in the Enkid study ${ }^{34}$. The index contains 16 elements and is composed of a scale from 0 to
12 points. A point was added if a series of Mediterranean characteristics were met, and subtracting a point with 'Westernised' or harmful food behaviours such as frequently consuming 'fast foods', pastries and sweets and not having breakfast. $4.2 \%$ of the sample presented a poor MDP, $49.4 \%$ had an intermediate pattern and $46.4 \%$ an excellent MDP. In high social classes there was a greater proportion of children and adolescents with excellent Mediterranean diets (54.9\%) as compared to lower (42.8\%) and medium (47.6\%) classes.

\section{Indexes based on the Mediterranean Diet Pyramid}

Mediterranean score. Canadian researchers studied 77 women to examine food habits, plasma lipoprotein profiles and body weight modifications based on a 12-week nutritional intervention promoting the $\mathrm{MD}^{35}$. Scoring based on 11 components of the Mediterranean pyramid by Oldways Preservation Trust was designed to evaluate MDP adherence. A partial score of 0 to 4 was attributed to each component. Food found at the base of the pyramid received a high score when consumed frequently. However, food found at the peak of the pyramid (meat, sweets and eggs) was given a high score when consumed less frequently. From the nutritional intervention, it was observed that the 'Mediterranean score' sample mean increased significantly from 21.1 points to 28 points, and resulted in a slight but significant improvement of the metabolic profile (total cholesterol, apoB levels and BMI), a significant decrease in energy coming from lipids and a significant reduction in weight and waist circumference.

Dietary score. Greek researchers constructed a dietary score also based on the Mediterranean Diet Pyramid ${ }^{11}$ to study the differences in plasma lipids according to MDP adherence $^{36}$. The index was constructed based on higher scoring from 0 to 5 points according to intake frequency of typical Mediterranean products, and for those components far from the MDP a decreased scoring. The adherence to the Mediterranean diet resulted in significant reductions only for levels of oxidised LDL-cholesterol.

\section{Mediterranean Diet Quality Index}

The Mediterranean Diet Quality Index (Med-DQI) was an adaptation of the 'Diet Quality Index'37 to evaluate the MDP. Olive oil, fish, and alternative meat substitutes were added $^{38}$. The objective of the study was to evaluate the adherence to the MDP in a French population, to study the socio demographic and lifestyle associated factors, and to validate and correlate the Med-DQI with biological markers (carotene, vitamin E, EPA and DHA). In the Med-DQI a score from 0 to 2 was assigned to each food group according to the recommendations when existing, or otherwise using the population intake tertiles to assign cut-off points. The total score of the index was from 0 to 14 points. The lower the Med-DQI value, the healthier the diet. Elder individuals and those living in rural areas, 
working class individuals and non-smokers presented a greater Med-DQI. There was a high correlation between Med-DQI and studied biomarkers, with the exception of cholesterol.

\section{Mediterranean diet pattern score}

In an Italian cohort study, a score was created to be able to study the association between the MDP and the risk of developing peripherical vascular disease in type II diabetic patients $^{39}$ as it was postulated that more than a specific food alone, a high score may play a protective role. A score was developed where a point was added according to food intake with sufficient evidence of its beneficial effect on coronary artery disease, and 0 for those foods that have potentially harmful effects. Specifically, the study found that individuals who obtained 11 points or more had $56 \%$ less risk of suffering this illness.

\section{Adberence index that adds and subtracts standardised components}

The SUN study is a prospective cohort study based on a sample of 4259 University of Navarra students with the objective of assessing the protective role of the MDP on coronary diseases and evaluating variables associated with this pattern ${ }^{40}$. An index was built that calculated a value of adherence as a percentage, standardising the food intake values to add and subtract the components depending on its nature. The results suggest that there is a progressive abandonment of the traditional MDP in younger individuals and those individuals who led an active lifestyle had greater adherence to the MDP.

In a transverse study of similar characteristics carried out in the Balearic Islands ${ }^{41}$, a variant of the Sanchez-Villegas index was applied. It also had the objective of analysing the prevalence of the MDP in a sample of 1200 individuals and the sociodemographic and lifestyle factors related to this pattern. The adherence to the MDP was defined through 9 points or characteristics, with small component modifications as compared to Sanchez-Villegas et al., but with the same calculation methodology. The MDP adherence in the Balearic population was $43.1 \%$, similar for all sociodemographic groups and lifestyles but with differences in age, sex, physical activity and smoking habits.

\section{Mediterranean Adequacy Index: quotient between components}

The adherence to the Italian MDP reference was measured in two Italian cohorts of the Seven Countries Study (Crevalcore and Montegiorgio) $^{42}$. The 'Mediterranean Adequacy Index' (MAI) was based on a quotient between the sum of energy proceeding from Mediterranean products (carbohydrate and protective food groups) and the sum of energy from non-Mediterranean products (animal origin foods and sweets). High index values indicated a greater MDP adherence. During the 31-year follow-up, a progressive abandonment of the MDP was observed, taking as a reference an Italian MDP from the town of Nicotera.

The MAI was also applied to Spanish Family Food Balance Sheets from the Ministry of Food and Agriculture $^{43}$. A total of 3022 Spanish households participated in the study to evaluate the influence of sociodemographic variables towards the adherence to the MDP. To apply MAI to Spanish data and to define Spanish MDP products a classification based on the Mediterranean Diet Pyramid was created, placing in the numerator those foods at the base of the pyramid and in the denominator those found at the vertex. Generally, high MAI values were observed in lower classes than in higher classes. Also, less adherence to the MDP was found in cities as compared to towns.

\section{Discussion}

Food pattern studies summarise the complexity of a diet taking into account the synergic effects or interactions between nutrients or foods that comprise the diet ${ }^{44,45}$.

Currently, indexes to measure the MDP are being explored in epidemiological studies, but not so much in experimental studies ${ }^{46}$. Simultaneously, there have been some attempts for outlining and specifying the definition of this food pattern ${ }^{3,10,11}$. According to the experts, there is still the need for a more precise and quantified definition of the pattern, which could be obtained, for example, by establishing inferior and/or superior component limits ${ }^{47}$. However, reaching a consensus is complex since the pattern may be related to a specific Mediterranean region and to a selected period that are adopted as references. Moreover, apart from using the traditional MDP as the baseline, current debates include the admission of a 'modern' MDP definition that incorporates current scientific knowledge on the relationship between diet and chronic diseases.

These problems with the definition of the pattern indirectly affect pattern evaluation methods and components used. For instance, there is a debate on the type of fat to be included in the definition (monounsaturated versus polyunsaturated) ${ }^{22}$, on how to include dairy products (their proportion and composition) ${ }^{21}$, the importance of different types of meat ${ }^{33}$, the classification of refined cereals as protective or 'non-protective' components $^{21}$, the establishment of a definition for moderate alcohol intake, and the presence of nuts and fish as independent components ${ }^{27}$.

On the other hand, Mediterranean diet evaluation methods that utilise scorings are limited by subjectivity in the selection of scoring components, mostly conditioned by available data and by the study's own objectives in assigning cut-off points and by the interpretation of the diet-disease relationship ${ }^{16}$. The variability in choosing cut-off points in the score, and distributing the population into different intake groups according to the grade of 
adherence, may influence the interpretation of results. Also, the use of indexes and cut-off points by other authors is a limitation since the population in which the index is applied may differ from the population for which the index was originally designed.

Furthermore, the majority of indexes do not really measure the adherence to a universal MDP but rather to a specific pattern, based on the distribution of selected food groups in the same population. This makes international comparisons more difficult. Some indexes postulate monotonic relations and do not take into account possible tolerance limits ${ }^{48}$. Another component-related problem is that the same importance is usually arbitrarily given to all index components, independently of the components' proportions in the diet and of the scientific evidence on the diet-disease relationship ${ }^{26}$.

Even though it is difficult to quantify the adherence to a diet and despite the fact that indexes require operational definitions, grouping foods to obtain complex scores is a very useful method to evaluate epidemiological associations $^{39}$. It is a simple and intuitive approximation to estimate attributable risk to a dietary pattern, although it does not result in such apparently strong associations as those obtained with a posteriori analytical methods ${ }^{49}$. Earlier studies have focused on the Mediterranean diet and life expectancy relationships within elderly populations: a 4-point increase in the MDS was associated with a significant reduction of total mortality in elderly in studies conducted in Greece, Denmark, Australia, Spain and throughout Europe ${ }^{15,23,28}$. In the last few years, however, age ranges of the studied populations have been amplified and the studied MDP-health associations expanded ${ }^{20}$. For instance, a 5-point increase in the MDS led to a reduction in $\mathrm{BMI}^{27}$. On the other hand, a 1-point reduction of the MDS was related to a significant increase in cardiovascular risk $^{32}$. A greater adherence to MDP led to a significant reduction in blood pressure ${ }^{21}$ and in the risk of aerodigestive tract cancers $^{8}$, of suffering an acute myocardial infarction $^{33}$ and of peripherical vascular disease in type II diabetes patients ${ }^{39}$. A simple nutritional intervention to promote the MDP may be effective in modifying nutritional habits in people in free-living conditions and may result in significant effects on apoB and BMI reduction ${ }^{35}$.

Some positive aspects of the reviewed studies can be found in the use of samples of extensive populations with a wide variety of patterns and lifestyles, and the use of valid frequency questionnaires ${ }^{22,28}$. Other elements that allow a critical evaluation of the MD-health hypothesis are the prospectiveness of the study, the evaluation of many confounding variables, the participation of cohorts from different European regions and the adjustment of dietary exposure factors between countries ${ }^{22}$. The Mediterranean diet adherence indexes are especially useful when the MD is compared with other food patterns, such as the Western pattern.
Some of the revised studies show problems related to dietary assessment methods. The majority of indexes are based on food groups, which require food categorisation, with the associated difficulties when evaluating mixed dishes.

The identified co-variables to be controlled when studying the MDP-health relations are also diverse in the different studies, basically influenced by their objectives. Age, sex, smoking and physical activity are the most frequently controlled variables because of their strong association to the MDP. To control for dieting is also considered to be necessary, since modifying food habits due to presence of disease may bring on increased MDP adherence ${ }^{25}$.

Other commonly controlled demographic variables are the geographic origin of the population, educational level, ethnic origin or social class. Among clinical and anthropometric variables are cholesterol, self-assessment of health status, mobility, blood pressure, diabetes and BMI.

In the different studies the MDP is analysed in several ways. Dietary indexes are being used to evaluate the degree of adherence to the MDP, to study the socioeconomic and lifestyle variables that influence the adherence to the pattern and to explore the multiple associations between the Mediterranean diet, as an integral entity, and health parameters such as life expectancy or the incidence of obesity, cardiovascular diseases and certain types of cancers. The evaluation of these associations is based on the risk reduction for chronic diseases, or on the modification of a clinical parameter of nutritional status (biochemical, anthropometric and clinical). Moreover, these indexes are also useful tools to measure food consumption trends and to identify the involved factors, as well as to develop comprehensive public health nutrition recommendations.

To sum up, a prudent dietary pattern for health promotion apparently coincides with the Mediterranean diet. MD indexes are useful tools to study this pattern and its association with health. A more precise and quantitative definition of the Mediterranean diet, however, is required if the adherence to such a dietary pattern is intended to be more accurately measured. Other aspects of the Mediterranean diet indexes should also be taken into account, such as the inclusion of typical Mediterranean foods like nuts and fish and the validation of the dietary pattern approach via the use of biomarkers.

\section{References}

1 Keys A, Menotti A, Karvonen M. The diet and 15-year death rate in the Seven Countries study. American Journal of Epidemiology 1986; 124: 903-15.

2 Nestle M. Mediterranean diets: historical and research overview. American Journal of Clinical Nutrition 1995; 61: 1313S-20S 
3 De Lorgeril M, Salen P, Martin J, Monjaud I, Delaye J, Mamelle N. Mediterranean diet, traditional risk factors and the rate of cardiovascular complications after myocardial infarction: final report of the Lyon Diet Heart Study. Circulation 1999; 99: 779-85.

4 Ryan M, McInerney D, Owens D, Collins P, Johnson A, Tomkin G. Diabetes and the Mediterranean diet: beneficial effects of oleic acid. Quarterly Journal of Medicine 2000; 93 : $85-91$.

5 Ferrara L, Raimondi S, D’Episcopo L, Guilda L, Dello Russo A, Marotta T. Olive oil and reduced need for antihypertensive medications. Archives of Internal Medicine 2000; 160: $837-42$

6 Joshipura KJ, Ascherio A, Manson JE, Stampfer MJ, Rimm EB, Speizer FE, et al. Fruit and vegetable intake in relation to risk of ischemic stroke. Journal of the American Medical Association 1999; 282: 1233-9.

7 Trichopoulou A, Georgiou E, Bassiakos Y, Lipworth L, Lagiou P, Proukakis C, et al. Energy intake and monounsaturated fat in relation to bone mineral density among women and men in Greece. Preventive Medicine 1997; 26: $395-400$.

8 Bosetti C, Gallus S, Trichopoulou A, Talamini R, Franceschi $\mathrm{S}$, Negri E, et al. Influence of the Mediterranean diet on the risk of cancers of the upper aerodigestive tract. Cancer Epidemiology, Biomarkers \& Prevention 2003; 12: 1091-4.

9 Matelas A, Zampelas A, Stavrinos V, Wolinky I. The Mediterranean Diet: Constituents and Health Promotion. Boca Raton, FL: CRC Press, 2000.

10 Serra-Majem L, Helsing E. Changing patterns of fat intake in Mediterranean countries. European Journal of Clinical Nutrition 1993; 47: 1S-100S.

11 Willett W, Sacks F, Trichopoulou A, Drescher G, Ferro-Luzzi A, Helsing E, et al. Mediterranean diet pyramid: a cultural model for healthy eating. American Journal of Clinical Nutrition 1995; 61: 1402S-6S.

12 WCRF. Nutrition and the Prevention of Cancer: A Global Perspective. Washington, DC: World Cancer Research Fund and American Institute for Cancer Prevention, 1997.

13 WHO. Diet, Nutrition and the Prevention of Chronic Diseases. Geneva: World Health Organization, 2003.

14 Hu F, Rimm E, Stampfer M. Prospective study of major dietary patterns and risk of coronary heart disease in men. American Journal of Clinical Nutrition 2000; 72: 912-21.

15 Trichopoulos D, Lagiou P. Dietary patterns and mortality. British Journal of Nutrition 2001; 85: 133-4.

16 Hu F. Dietary pattern analysis: a new direction in nutritional epidemiology. Current Opinion in Lipidology 2002; 13: 3-9.

17 Trichopoulou A, Kouris-Blazos A, Wahlquivist M, Gnardellis D, Lagiou P, Polychronopoulos E, et al. Diet and overall survival in elderly people. British Medical Journal 1995; 311: $1457-60$.

18 Costacou T, Bamia C, Ferrari P, Riboli E, Trichopoulos D, Trichopoulou A. Tracing the Mediterranean diet through principal components and cluster analyses in the Greek population. European Journal of Clinical Nutrition 2003; 57: $1378-85$.

19 Osler M, Schrool M. Diet and mortality in a cohort of elderly people in a north European community. International Journal of Epidemiology 1997; 26: 155-9.

20 Trichopoulou A, Costacou T, Bamia C, Trichopoulos D. Adherence to a Mediterranean diet and survival in a Greek population. New England Journal of Medicine 2003; 348: 2599-608.

21 Psaltopoulou T, Naska A, Orfanos P, Trichopoulos D, Mountokalakis T, Trichopoulou A. Olive oil, the Mediterranean diet, and arterial blood pressure: the Greek European Prospective Investigation into Cancer and Nutrition (EPIC) Study. American Journal of Clinical Nutrition 2004; 80: 1012-18.
22 Trichopoulou A, Bamia C, Trichopoulos D. Mediterranean diet and survival among patients with coronary heart disease in Greece. Archives of Internal Medicine 2005; 165: 929-35.

23 Kouris-Blazos A, Gnardellis C, Wahlqvist M, Trichopoulos D, Lukito W, Trichopoulou A. Are the advantages of the Mediterranean diet transferable to other populations? A cohort study in Melbourne, Australia. British Journal of Nutrition 1999; 82: 57-61.

24 Woo J, Woo K, Leung S. The Mediterranean score of dietary habits in Chinese populations in four different geographical areas. European Journal of Clinical Nutrition 2001; 55: $215-20$

25 Lasheras C, Fernandez S, Patternson. Mediterranean diet and age with respect to overall survival in institutionalised, nonsmoking elderly people. American Journal of Clinical Nutrition 2000; 71: 987-92.

26 González C, Argílaga S, Agudo A, Amiano P, Barricarte A, Beguiristain JM, et al. Sociodemographic differences in adherence to the Mediterranean dietary pattern in Spanish populations. Gaceta Sanitaria 2002; 16: 214-21.

27 Schröder H, Marrugat J, Vila J, Covas M, Elosua R. Adherence to the traditional mediterranean diet is inversely associated with body mass index and obesity in Spanish population. Journal of Nutrition 2004; 134: 3355-61.

28 Knoops K, De Groot L, Kromhout D, Perrin A, MoreirasVarela $\mathrm{O}$, Menotti A, et al. Mediterranean diet, lifestyle factors, and 10-year mortality in elderly European men and women. Journal of the American Medical Association 2004; 292: $1433-9$.

29 Van Staveren W, De Groot L, Haveman-Nies A. The SENECA study: potentials and problems in relating diet to survival over 10 years. Public Health Nutrition 2002; 5: 901-5.

30 Trichopoulou A, Orfanos P, Norat T, Bueno-de-Mesquita B, Ooke MC, Peeters PH, et al. Modified Mediterranean diet and survival: EPIC-elderly prospective cohort study. British Medical Journal 2005; 330: 991-7.

31 Trichopoulos D, Lagiou P. Mediterranean diet and overall mortality differences in the European Union. Public Health Nutrition 2004; 7: 949-51.

32 Bilenko N, Fraser D, Vardi H, Shai I, Shahar D. Mediterranean diet and cardiovascular diseases in an Israeli population. Preventive Medicine 2005; 40: 299-305.

33 Martínez-González M, Fernández-Jarne E, Serrano-Martínez M, Marti A, Martínez J, Martín-Moreno J. Mediterranean diet and reduction in the risk of a first acute myocardial infarction: an operational healthy dietary score. European Journal of Nutrition 2002; 41: 153-60.

34 Serra-Majem L, Ribas L, Ngo J, Ortega R, García A, PérezRogrigo C, et al. Food youth and the Mediterranean diet in Spain. Development of KIDMED, Mediterranean Diet Quality Index in children and adolescents. Public Health Nutrition 2004; 7: 931-5.

35 Goulet J, Lamarche B, Nadeau G, Lemieux S. Effect of a nutritional intervention promoting the Mediterranean food pattern on plasma lipids, lipoproteins and body weight in healthy French-Canadian women. Atherosclerosis 2003; 170: $115-24$

36 Panagiotakos D, Pitsavos C, Polychronopoulos E, Chrysohoou C, Zampelas A, Trichopoulou A. Can a Mediterranean diet moderate the development and clinical progression of coronary heart disease? A systematic review. Medical Science Monitor 2004; 10: 193-8.

37 Patterson RE, Haines PS, Popkin BM. Diet quality index: capturing a multidimensional behaviour. Journal of the American Dietetic Association 1994; 94: 57-64.

38 Gerber M. Qualitative methods to evaluate Mediterranean diet in adults. Public Health Nutrition 2006; 9 (1A): 147-51.

39 Ciccarone E, Di Castelnuovo A, Salcuni M, Siani A, Giacco A, Donati M, et al. A high-score Mediterranean dietary pattern is associated with a reduced risk of peripheral arterial disease 
in Italian patients with type 2 diabetes. Journal of Thrombosis and Haemostasis 2003; 1: 1744-52.

40 Sanchez-Villegas A, Martinez J, de Irala J, Martínez-González M. Determinants of the adherence to an 'a priori defined Mediterranean dietary pattern. European Journal of Nutrition 2002; 41: 249-57.

41 Tur J, Romaguera D, Pons A. Adherence to the Mediterranean dietary pattern among the population of the Balearic Islands. British Journal of Nutrition 2004; 92: 341-6.

42 Alberti-Fidanza A, Fidanza F, Chiuchiu M, Verducci G, Fruttini D. Dietary studies on two rural Italian population groups of the Seven Countries Study. 3. Trend of food and nutrient intake from 1960 to 1991. European Journal of Clinical Nutrition 1999; 53: 854-60.

43 Fuentes-Bol M. La dieta mediterránea: su evolución. In: Serra-Majem L, Ngo J, eds. ¿Qué es la Dieta Mediterránea? Fundación para el desarrollo de la Dieta Mediterránea. Barcelona: Nexus Editions, 2002; 60-73.

44 Kant A. Dietary patterns and health outcomes. Journal of the American Dietetic Association 2004; 104: 615-36.
45 Hu F. The Mediterranean diet and mortality - olive oil and beyond. New England Journal of Medicine 2003; 348 2595-6.

46 Serra-Majem L, Roman B, Estruch R. Scientific evidence of interventions using the Mediterranean diet: a systematic review. Nutrition Reviews 2006; 64: 531-52.

47 Serra-Majem L, Trichopoulou A, Ngo J, Cervera P, García A, La Vecchia C, et al. Does the definition of the Mediterranean diet need to be updated? Public Health Nutrition 2004; 7: 927-9

48 Trichopoulou A, Naska A, Antoniou A, Friel S, Trygg K, Turrini A. Vegetable and fruit: the evidence in their favour and the public health perspective. International Journal for Vitamin and Nutrition Research 2003; 73: 63-9.

49 Jacques P, Tucker K. Are dietary patterns useful for understanding the role of diet in chronic disease? European Journal of Clinical Nutriton 2001; 73: 1-2.

50 Alberti-Fidanza A, Findanza F. Mediterranean Adequacy Index of Italian diets. Public Health Nutrition 2004; 7: 937-41. 